\title{
Broad-Time-Horizon Solar Power Prediction and PV Performance Degradation Research at the University of Arizona
}

\author{
B.G. Potter, Jr., Kelly Simmons-Potter, William F. Holmgren \\ University of Arizona, Tucson, AZ, 85721, USA
}

\begin{abstract}
An overview of University of Arizona cooperative research efforts towards enhanced solar power prediction over PV system operational lifetime is provided. Integration of established research programs in power forecasting (including irradiance and irradiance-to-power modeling) and in device and system-level performance degradation studies offer new opportunities to address solar power prediction needs over shortand long-term time horizons.
\end{abstract}

Index Terms - solar power generation, forecasting, degradation, photovoltaic cells

\section{INTRODUCTION}

Effective integration of renewable energy for grid-based application requires varied time-horizon prediction of anticipated load and generation capacity. In Arizona alone, utility companies, homeowners, and third-party developers have installed over 3000 MW of solar power, and an additional $3800 \mathrm{MW}$ of solar power is expected to be installed over the next 5 years [1]. A key challenge to economically managing and developing the growing solar energy resource is the accurate prediction of solar power plant short-term energy yield (driven by weather and irradiance variability) and the long-term energy yield (driven by system degradation). The University of Arizona (UA) Institute for Energy Solutions integrates core competencies spanning atmospheric forecasting, wind and solar irradiance prediction, multitechnology PV power generation, and module performance degradation assessment to address challenges to effectively manage an increasingly heterogeneous power generation portfolio over near and long-term time scales. UA's Power Forecasting Group pursues fundamental research and develops, and operationally produces solar and wind power forecasts for four electric utility companies in the Western U.S. (Tucson Electric Power, Arizona Public Service, Public Service Company of New Mexico, and Idaho Power Company). In addition, the Depts. of Electrical and Computer Engineering and Materials Science and Engineering, through the Arizona Research Institute for Solar Energy (AzRISE) have established facilities to pursue irradiance-to-power characterization and modeling, full-scale accelerated-lifecycle testing of solar modules and battery storage, and field-based module performance analysis at the Tucson Electric Power (TEP)-AzRISE solar test yard. The integration of these programs offers a unique assembly of enabling technologies and expertise that is focused on increasing the fidelity and application range of renewable energy power generation forecasting, combining both increased conventional forecast accuracy with enhanced site- and technology-specific prediction for long-term generation output and future plant planning.

\section{FACILITIES AND BACKGROUND}

The UA Power Forecasting Group (UA-PFG) generates forecasts of weather, solar power, and wind power on time horizons from minutes to 7 days. A combination of real-time solar power data from utility-scale plants and rooftop PV, geostationary satellite imagery, and a $1.8 \mathrm{~km}$ resolution configuration of the Weather Research and Forecasting model is used to achieve better forecast accuracy at different time horizons [2-4]. Recent efforts have focused on the development of a new data assimilation methodology using a distributed sensor and PV generation network to increase local irradiance forecasting accuracy [4].

In addition to irradiance forecast uncertainty, a major deficiency of the solar power forecasts is an overly-simplified irradiance-to-power (I-P) algorithm and, in the longer term, no inclusion of the effects of system degradation on I-P model parameters. The UA AzRISE team has developed a range of facilities and expertise to address performance testing and environmental degradation phenomena in both laboratory and field contexts. Efforts span material-, cell-, and module-level studies to provide insight into the effect of design and operational conditions (e.g. spectral irradiance, incidence angle) and time-dependent degradation on PV system output.

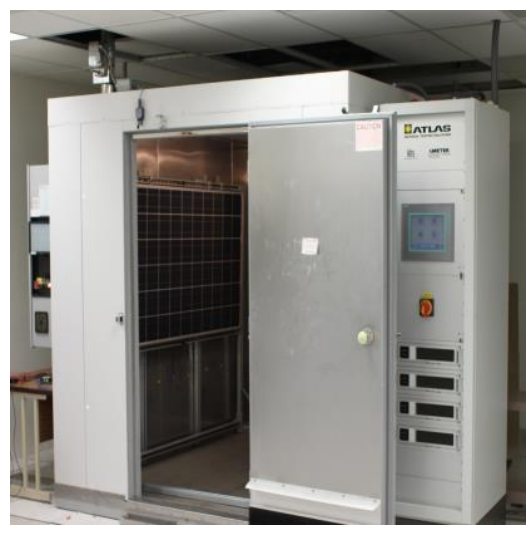

Fig. 1. Large-scale PV module environmental test chamber at UA. 
Primary facilities include a large-scale, walk-in accelerateddegradation chamber (Figure 1) with temperature control over $-30^{\circ} \mathrm{C}$ to $85^{\circ} \mathrm{C}$, humidity control up to $95 \%$ relative humidity, uniform full solar irradiance in excess of $1000 \mathrm{~W} / \mathrm{m}^{2}$ over a 2 $\mathrm{m} \times 1.3 \mathrm{~m}$ area, and in-situ monitoring of module performance (including I-V and electroluminescence (EL) [5]). The chamber is used routinely for accelerated lifecycle (ALC) studies and is also equipped for IEC-compliant qualification testing.

The environmental chamber is complemented by the TEPAzRISE solar test yard (Figure 2). This facility provides for installation and testing of inverter and module technologies. Capabilities include measurement of AC power, DC power, local irradiance and ambient temperature, as well as back-side temperature on selected PV module technologies.

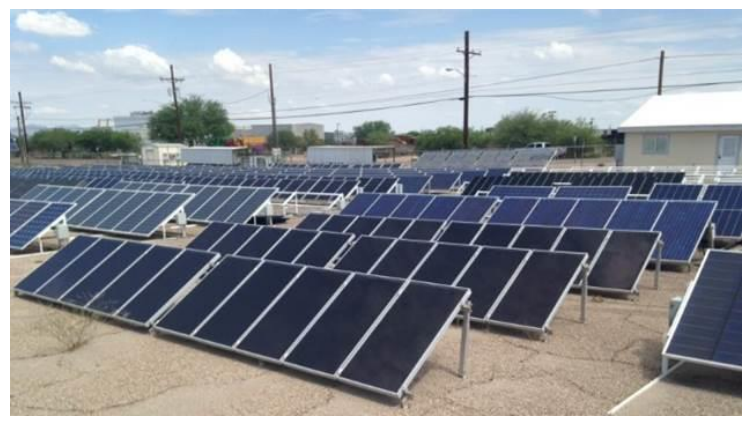

Fig. 2. Strings of PV modules at TEP/AzRISE test yard in Tucson, AZ.

The test yard has undergone extensive electronics and data technology infrastructure refurbishment over the past year to provide high-reliability, module and string performance data and weather condition metrics with redundant data collection and online data access [6]. Examples of data, collected from a string of poly-Si PV modules on one minute intervals, are shown below.

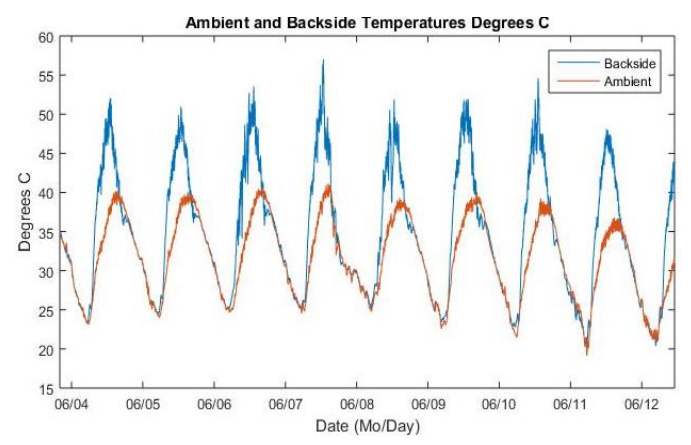

Fig 3. Comparison of ambient air temperature with PV module backside temperature data collected at TEP/AzRISE test yard.
Figure 3 compares backside PV module temperature, taken by averaging data from three T-type thermocouples affixed to the rear side of several representative poly-Si PV modules, to ambient temperatures obtained from a weather station that is located at the test yard. Figure 4 shows the measurement of DC voltage on a poly-Si PV module string over the course of one week. Evident in Fig. 4 is the expected dip in PV power output due to increased mid-day ambient temperatures, as well as the effects of daily morning cloud cover experienced at the test yard during the week depicted.

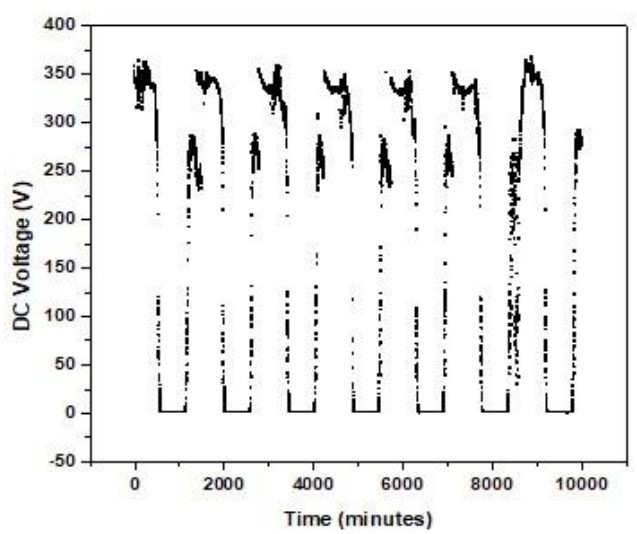

Fig. 4. DC power generated by a PV module string at the TEP/AzRISE test yard.

To assist with both irradiance forecast model validation and I-P model assessment, the Solar Field (SOFIE) autonomous irradiance measurement station is GPS-enabled and measures irradiance (DNI, GHI, DHI, POA), wind speed, wind direction, and air temperature (Figure 5). In a previous effort, SOFIE was deployed for over 16 months of continuous operation at the SunEdison Picture Rocks solar power plant (25MW). SOFIE is currently located at the TEP/AzRISE test yard in Tucson, AZ.

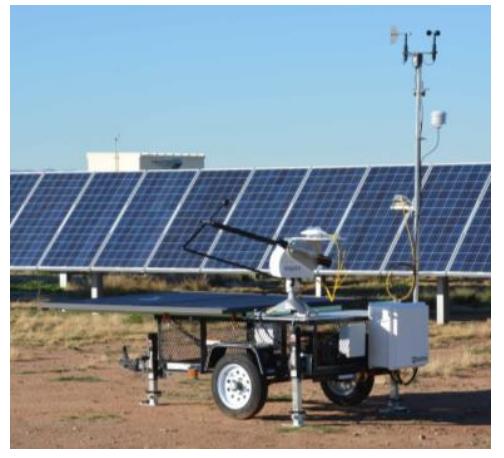

Fig. 5. SOFIE autonomous irradiance and weather measurement station. 


\section{RESULTS}

Examples of power forecasting and PV performance and degradation assessment results provide a context for new efforts to leverage these capabilities toward the improvement of solar forecast model development and its application for longer time-frame predictions.

In past work, UA's PFG has led the development of technology and methods to improve satellite-derived irradiance forecasts using a sparse set of readily deployed sensor elements and distributed PV installations. Using a Bayesian technique (optimal interpolation (OI)), significant improvement in GHI nowcasts can be achieved under a variety of weather conditions [4,7]. As shown in Figure 6, the use of sparse ground data and OI methodology (associated with "sensor" legend items in Figure 6) resulted in a significant improvement in GHI prediction compared with a prediction that did not involve OI (labelled "Background" in the Figure). Up to a $50 \%$ reduction in GHI RMSE was obtained. We are now researching how to extend the OI framework to DNI and POA using data from the SOFIE platform.

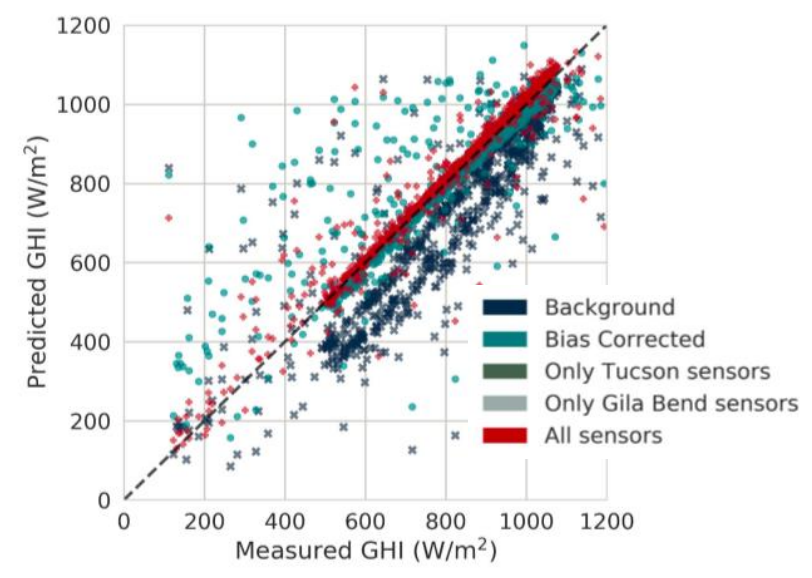

Fig. 6. GHI predicted and measured results showing impact of OI-enhanced model involving sparse ground-based sensors.

Ensemble forecast systems have been shown to be powerful tools in the weather forecast community. UA is developing new models for intrahour ensemble irradiance forecasts [8]. The model uses the local ensemble transform Kalman filter (LETKF), a data assimilation technique, to combine ground data, satellite images, satellite-derived winds using optical flow, and winds from a full weather model (WRF) (see Figure 7). The resulting forecasts are more accurate than those from any one of the constituent data sources, and the forecast ensemble provides an estimate of forecast uncertainty. Importantly, the forecast system can be run in real-time on a single multi-core computer, rather than on a more expensive high-performance computing center. Further work is needed to better quantify the relative accuracy of the simplified ensemble forecast versus full-featured deterministic weather models, however, preliminary results suggest the LETKFbased technique is competitive for intrahour forecasts.
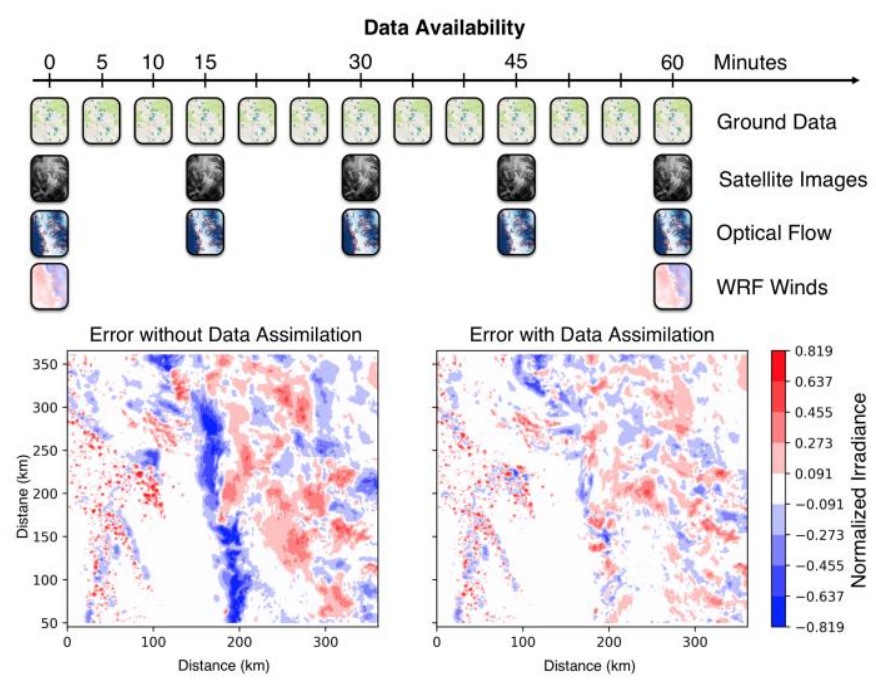

Fig. 7. Top: Forecast data sources and the times at which they are assimilated into the LETKF ensemble forecast system. Bottom: Map showing the reduction in mean irradiance forecast error using the LETKF data assimilation.

Efforts by the UA team in irradiance-to-power model assessment and implementation address additional enhancement in short-term power forecasting. For example, UA's AzRISE team has examined the potential to integrate technology-specific device response functions into established I-P models. These efforts can offer power generation prediction both for existing facilities and for new installations specific to anticipated deployment and technology conditions [9]. Figure 8 shows the use of incident-angle dependent EQE data to inform $\mathrm{f}_{2}$, a key incidence angle parameter in the Sandia Array Performance Model (SAPM). In addition, onsite access to irradiance information via SOFIE has provided additional insight into

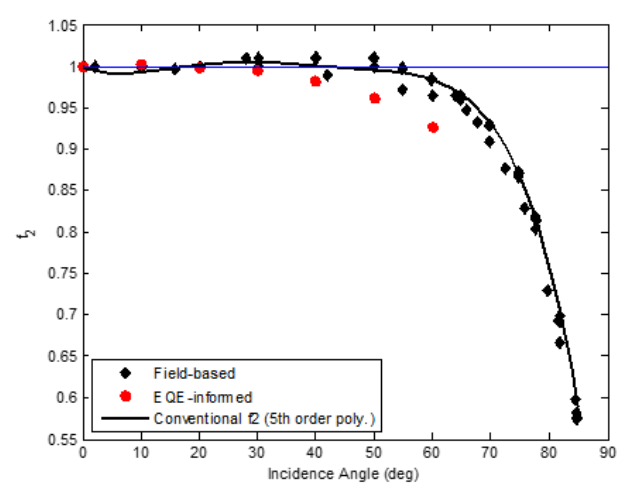

Fig. 8. The SAPM incidence angle functions determined by field-based parameter fitting and EQE-informed methods. 
opportunities for improved I-P (PVLib) model performance [10].

UA is also teaming with the PV modeling community to promote the use and development of open source PV modeling tools (PVLib [11] and others [12]) as well as the development of new assessment frameworks for predictive models [13].

In the context of longer time-horizon power forecasting, environmental degradation in power conversion efficiency can significantly undermine the long-term performance and hence the lifetime of photovoltaic (PV) modules. Moreover, manufacturer-supplied ratings (such as efficiency derating estimations) do not address nonstandard climate conditions. These limitations can significantly impact the optimization of predictive models relating irradiance-to-power. Integration of time-dependent parameter information unique to the module, for example, can assist in the more rapid validation of power forecast models over the PV system lifetime. The UA team has used accelerated and field-relevant module and string-level testing facilities to evaluate such long-term performance degradation.

To-date, multiple Si-based technologies and CdTe thin film systems subjected to accelerated lifecycle (ALC) testing conditions that mimic weather and irradiance conditions at the TEP-AzRISE test yard have confirmed the viability of the UA ALC test method in predicting performance efficiency degradation while demonstrating a $3.6 \mathrm{X}$ decrease in assessment time compared with field studies [14]. Figure 9 shows representative ALC data obtained for a multicrystalline Si PV module. An experimentally determined temperature derating has been removed to isolate time-dependent, in-situ performance degradation. Such data can be used to tune I-P model parameters providing consistent power prediction performance over the system life.

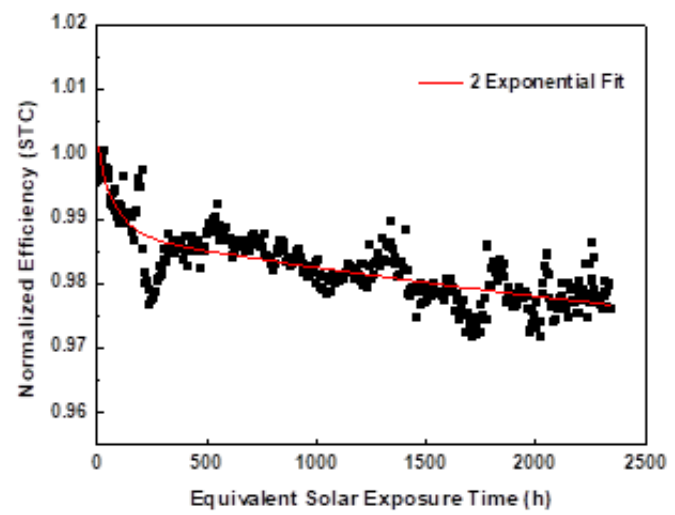

Fig. 9. Normalized energy conversion efficiency for a multicrystalline Si PV module under accelerated lifecycle test conditions (simulated time period $=$ one year).

\section{CONCLUSION}

Enhanced reliability of PV energy generation prediction over the system lifetime requires the integration of competencies and facilities to examine a broad range of coupled considerations, including irradiance forecasting, irradiance-to-power modeling and longer time-horizon system performance degradation. The University of Arizona plays a unique role in this multidisciplinary effort, combining expertise in predictive model development that addresses atmospherics, irradiance, and irradiance-to-power with materials and system degradation assessment to offer capability development opportunities of direct impact to utility-level energy generation, grid integration, and technology improvement.

The authors thank the University of Arizona Institute for Energy Solutions, Tucson Electric Power, Arizona Public Service, and Public Service Company of New Mexico for support.

\section{REFERENCES}

[1] SEIA 2018, https://www.seia.org/state-solarpolicy/arizona-solar, accessed 1/21/2018.

[2] Lorenzo et. al., "Irradiance forecasts based on an irradiance monitoring network, cloud motion, and spatial averaging," Solar Energy, 122, 1158 (2015).

[3] Kim et. al., "Toward Improved Solar Irradiance Forecasts: Derivation of Downwelling Surface Shortwave Radiation in Arizona from Satellite," Pure and Applied Geophysics, 173, 2535 (2016).

[4] Lorenzo et. al., "Optimal interpolation of satellite and ground data for irradiance nowcasting at city scales," Solar Energy, 144, 466 (2017).

[5] T. Lai, et. al., "Image analysis of PV module electroluminescence," Proc. SPIE, Vol. 10370, pp. 103700K-1 - 103700K-9, 2017.

[6] W. Bennett, et. al., "Data acquisition and PV module power production in upgraded TEP/AzRISE solar test yard," Proc. SPIE, Vol. 10370, pp. 103700G-1 103700G-7, 2017.

[7] Lorenzo et. al., "Data assimilation of rooftop solar power data to improve satellite derived nowcasts" $98^{\text {th }}$ Annual Meeting of the American Meteorological Society, 2018.

[8] Harty et. al., "Irradiance Forecasting Using the Local Ensemble Transform Kalman Filter, Satellite Images, and Ground Sensors" 98 $8^{\text {th }}$ Annual Meeting of the American Meteorological Society, 2018.

[9] Potter et. al., "Incidence-angle dependent external quantum efficiency: Laboratory characterization and use in irradiance-to-power modeling," 42nd IEEE Photovoltaic Specialist Conference, PVSC 2015. DOI: 10.1109/PVSC.2015.7355998. 
[10] Cuiffi et. al., "Ground-based irradiance measurement station for improved solar electrical production forecasting," SPIE Optics and Photonics; Conf. 9938, 2016.

[11] Stein et. al., "PVLIB: Open Source Photovoltaic Performance Modeling Functions for Matlab and Python" $43^{\text {rd }}$ Photovoltaics Specialists Conference, 2016.

[12] Holmgren et. al., "Review of open source tools for PV modeling" $45^{\text {th }}$ Photovoltaics Specialists Conference, 2018; http://github.com/wholmgren/openpvtools
[13] Holmgren et. al., "A comparison of PV power forecasts using PVLib Python" 44 ${ }^{\text {th }}$ Photovoltaics Specialists Conference, 2017.

[14] Lai et. al., "Analysis of twelve-month degradation in three polycrystalline photovoltaic modules," Proc. SPIE 9938, Reliability of Photovoltaic Cells, Modules, Components, and Systems IX, 993800 (26 September 2016); doi: 10.1117/12.2237527. 